\section{Análise de programas de preservação da audição em quatro indústrias metalúrgicas de Piracicaba, São Paulo, Brasil}

\author{
Program for hearing loss prevention \\ in four metallurgical factories in Piracicaba, \\ São Paulo, Brazil
}

\footnotetext{
1 Faculdade de Ciências da Saúde, Universidade Metodista de Piracicaba, Piracicaba, Brasil.

2 Faculdade de Ciências Médicas, Universidade Estadual de Campinas, Campinas, Brasil.

Correspondência C. G. O. Gonçalves Faculdade de Ciências da Saúde, Instituição: Universidade Metodista de Piracicaba. Rua Idelfonso Stehle 27, apto. 24, Indaiatuba, SP 13333-030, Brasil. cggoncal@unimep.br
}

\section{Abstract}

This study analyzes noise control programs in four metallurgical factories in Piracicaba, São Paulo, Brazil. From 1997 to 2001, 741 workers were examined and the programs were analyzed based on workers' profiles (2,270 audiometric exams) and documents on hearing preservation programs. $41 \%$ of workers presented hearing loss (mean age 42.3; mean years of work 16.7). 104 workers with more than one audiometric exam presented changes in hearing threshold. 36.5\% (38 workers) developed noise-induced hearing loss (NIHL) and 63.5\% (66 workers) with existing NIHL showed worsening of their hearing threshold. In these factories, $69.5 \%$ of workers were exposed to $\geq 84 d B(A)$. We concluded that the hearing preservation programs are not adequately organized and that workers are still exposed to high noise levels at work, posing a risk for NIHL. In addition, the current legislation on hearing loss prevention is not enforced.

Occupational Exposure; Noise-Induced Hearing Loss; Occupational Health; Occupational Health Program
Cláudia Giglio de Oliveira Gonçalves 1 Aparecida Mari Iguti 2

\section{Introdução}

A Consolidação das Leis Trabalhistas (CLT) rege as relações de trabalho entre empregados e empregadores e também sobre as condições de trabalho. As Normas Regulamentadoras (NR), em suas normatizações, estabelecem os parâmetros para a aplicação das leis. A NR-15 1 em seu Anexo I, estabelece os limites de tolerância para os trabalhadores expostos ao ruído, e a NR-7 2, a obrigatoriedade da elaboração e implementação do Programa de Controle Médico de Saúde Ocupacional (PCMSO), objetivando a preservação da saúde do conjunto dos trabalhadores. No seu Quadro II, estão os parâmetros para a monitorização da exposição ocupacional a agentes de risco à saúde, incluindo o ruído. Em seu Anexo II, constam as diretrizes e parâmetros mínimos para a avaliação e acompanhamento da audição em trabalhadores expostos a níveis de pressão sonora elevados e os subsídios para a adoção de programas de preservação da saúde auditiva dos trabalhadores, inclusive os parâmetros para a realização dos exames audiométricos e sua interpretação. Define também a aptidão ao trabalho, sugerindo que a Perda Auditiva Induzida pelo Ruído (PAIR) por si só não indica inaptidão ao trabalho e que diversos fatores devem ser considerados e, entre eles, a demanda auditiva para a função do trabalhador.

O Comitê Nacional de Ruído e Conservação Auditiva 3 lançou no Brasil o Boletim n. 6, as di- 
retrizes básicas de um Programa de Conservação da Audição (PCA), com recomendações mínimas para a sua elaboração, contendo as seguintes etapas: reconhecimento e avaliação de riscos para a audição, gerenciamento audiométrico, medidas de proteção coletivas, medida de proteção individual, educação e motivação, gerenciamento dos dados e avaliação do programa.

A proposta da American Academy of Otolaryngology (AAO) 4 inclui: medição e análise da exposição do trabalhador ao ruído, controle do ruído por medidas coletivas e individuais, instrução e motivação dos empregados às práticas de conservação auditivas, avaliação da audição dos trabalhadores e a avaliação e eficiência do programa. Na década de 90, os programas preventivos foram amplamente discutidos e órgãos americanos, como por exemplo a National Institute for Occupation Safety and Health (NIOSH), 5 estabeleceram uma revisão nas diretrizes para um PCA, incluindo orientações para avaliar-se a eficácia e eficiência do programa.

Todas essas diretrizes são justificáveis uma vez que a exposição ao ruído atinge uma grande parte da população trabalhadora em todo o mundo. O NIOSH 6 calculou a existência de 30 milhões de trabalhadores expostos a ruído nos Estados Unidos, o que significaria $26 \%$ da força produtiva do país.

Na Europa, estima-se que em torno de 30 milhões de pessoas trabalham em ambientes ruidosos e 10 milhões estão também expostas a agentes químicos ototóxicos 7 .

A exposição ao ruído intenso por períodos prolongados leva a efeitos auditivos (perdas auditivas) e aos não-auditivos, como alterações no sistema neurológico, no aparelho circulatório, aparelho digestivo, sistema endócrino, sistema imunológico e no psiquismo 8 .

A PAIR foi considerada na década de 80 a doença profissional mais freqüente no mundo todo e, na década de 90, mesmo com o despontamento das doenças músculo-esqueléticas, ainda esteve entre os principais problemas de saúde dos trabalhadores.

No Reino Unido, a PAIR foi a segunda doença do trabalho mais freqüente registrada oficialmente em 1990/1991 9

Na América Latina não foi diferente, como por exemplo na Venezuela, entre as doenças ocupacionais registradas no seguro social do país, a PAIR ocupou em 1994 o quarto lugar e o terceiro em 199510.

Lee-Feldstein 11 analisou a audição de 11.435 trabalhadores de uma indústria americana metalúrgica de grande porte, todos do sexo mas- culino e expostos a níveis de ruído superiores a $85 \mathrm{~dB}(\mathrm{~A})$, encontrando 5.814 (50,8\%) exames auditivos com indicativos de alterações. E Horg \& Raymond 12 avaliaram a audição de 575 trabalhadores expostos a ruído, com média de idade de 43 anos, e encontraram $60 \%$ destes com PAIR, sendo que $37 \%$ foram perdas auditivas moderadas ou severas.

No Brasil a situação nas últimas décadas não é diferente. Andrade \& Schochat 13 avaliaram 7.403 trabalhadores expostos a níveis de pressão sonora maiores que $85 \mathrm{~dB}(\mathrm{~A})$, provenientes de diversos ramos de atividades na Cidade de São Paulo, e encontraram alterações auditivas em trabalhadores nos seguintes ramos de atividade: pedreira $(54,4 \%)$; moinho e farinha $(37,1 \%$ de portadores de PAIR); metalurgia $(34,5 \%)$; vidros $(32,7 \%)$; tecelagem $(30,3 \%)$; papel e celulose $(30,2 \%)$.

Costa 14 avaliou a audição de 714 metalúrgicos com menos de dez anos de exposição a ruído, pertencentes a três indústrias no interior de São Paulo, e encontrou 22,9\% de traçados audiométricos compatíveis com PAIR. Kwitko \& Pezzi 15 analisaram a audição de 524 trabalhadores de indústrias metalúrgicas de Porto Alegre, Rio Grande do Sul, e encontraram 246 $(46,9 \%)$ trabalhadores com PAIR.

Franco 16 analisou a audição de 3.117 candidatos a empregos em diferentes empresas da região de Campinas, São Paulo, sendo 2.462 homens e 655 mulheres, com idades variando de 18 a 70 anos. Encontrou 610 (19,6\%) sujeitos com alterações auditivas. No Programa de Saúde do Trabalhador de Campinas, as estatísticas mostram que a PAIR ocupou o primeiro lugar entre as doenças ocupacionais diagnosticadas, representando $35,7 \%$ dos atendimentos em 1991 e 37,2\% em 199217.

Carneiro et al. 18 analisaram a audição de 3.750 trabalhadores expostos a ruído, todos homens com idade entre 20 e 60 anos, e encontraram $40 \%$ deles com audiograma alterado, sendo que $76 \%$ eram alterações auditivas bilaterais.

O objetivo deste estudo é analisar os programas de preservação da audição em quatro metalúrgicas de Piracicaba, São Paulo, no período de 1997 a 2001.

\section{Fontes de dados e método}

Com base nas informações sobre as PAIR notificadas por meio de Comunicações de Acidentes de Trabalho (CAT) no município, foram selecionadas as empresas com maior ocorrência de casos de PAIR em 1997/1998 e enviado um folheto contendo informações sobre a PAIR, o 
PCA e a proposta de um estudo. Daquelas empresas que manifestaram interesse em que fosse desenvolvido esse estudo (em um total de nove), após um contato inicial para caracterizá-las, quatro delas foram selecionadas. São empresas nacionais, três delas vinculadas ao setor sucroalcooleiro, pois a principal atividade agrícola da região é o plantio de cana-de-açúcar, e que já estão instaladas há muitos anos na cidade (uma delas foi a primeira metalúrgica a funcionar no município).

A população total estudada foi de 741 trabalhadores. As fontes de dados sobre a avaliação auditiva destes trabalhadores foram os prontuários ou os bancos de dados das empresas. No período de 1997 a 2001 foram levantados os Mapas de Agentes de Risco, as Avaliações Ambientais e de Ruído, os PCMSO e os Programas de Prevenção de Riscos Ambientais (PPRA). Também foram observados os sistemas de produção do trabalho por considerar que podem alterar os riscos a que os trabalhadores estão expostos.

Descreveu-se: o perfil auditivo dos trabalhadores segundo idade e tempo de serviço, o monitoramento auditivo, os níveis de pressão sonora em ambientes de trabalho e as medidas adotadas para o controle do ruído. Como critério para a análise dos exames audiométricos dos trabalhadores adotou-se a NR-7, Anexo II. A classificação foi: dentro dos limites aceitáveis, PAIR e perda auditiva não-ocupacional. Para a análise estatística dos dados coletados utilizou-se o programa SAS (SAS Institute, Cary, Estados Unidos). Realizou-se testes paramétricos, como teste $t$ de Student para a comparação entre as variáveis: empresa estudada e médias de idade e de tempo de serviço (parâmetros) dos trabalhadores; teste para análise de variância (ANOVA), associando mais de duas médias (as de idade e tempo de exposição ao ruído dos trabalhadores) entre as empresas es- tudadas e teste de Scheffé para identificação de semelhanças e dessemelhanças entre as médias de idade e tempo de serviço exposto ao ruído dos trabalhadores nas empresas. Testes não-paramétricos: qui-quadrado para verificação da associação entre as variáveis idade, tempo de serviço e deslocamento no limiar padrão.

\section{Resultados}

Algumas características gerais das quatro empresas estão na Tabela 1.

As empresas possuem certificação internacional para a sua produção (ISO 9000 ou 9002), e em três delas foram implantados sistemas de produção utilizando alguns elementos do Modelo Toyotista como "just-in-time", produção em células e no controle de qualidade. O ruído intenso é o principal fator de alterações auditivas e presente em grande parte das indústrias metalúrgicas.

As empresas estudadas consideram que o ruído é o principal agente de risco que possuem. Os Níveis de Pressão Sonora (NPS) aos quais os trabalhadores (741) estão expostos em seus postos de trabalho nas empresas foram analisados (Tabela 2).

Os NPS encontrados nos postos de trabalho são de $65 \mathrm{~dB}(\mathrm{~A})$ a $105 \mathrm{~dB}(\mathrm{~A})$, mensurados por leitura instantânea (utilizando equipamento medidor de pressão sonora) pelas próprias empresas ou por serviços contratados, não sendo realizados os cálculos de dose equivalente de exposição ao ruído. $30,5 \%$ de trabalhadores estão expostos a NPS entre 65 e $83 \mathrm{~dB}(\mathrm{~A})$, e os demais $(69,5 \%)$ encontram-se em níveis de ruído superiores a $84 \mathrm{~dB}(\mathrm{~A})$, configurando situação de risco à saúde desses trabalhadores. A média dos NPS por postos de trabalho é de $86,6 \mathrm{~dB}(\mathrm{~A})$ com desvio padrão de 6,07. A Empresa 3 apre-

Algumas características das quatro empresas estudadas.

\begin{tabular}{lcccc}
\hline & Empresa 1 & Empresa 2 & Empresa 3 & Empresa 4 \\
\hline Ano de fundação & 1973 & 1961 & 1925 & 1918 \\
Grau de risco & 3 & 3 & 3 & 3 \\
Sistema da qualidade & ISO 9000 & ISO 9002 & ISO 9002 & ISO 9002 \\
Sistema de produção & Just-in-time, & Misto & Just-in-time, & Tradicional \\
layout celular & & $32.822 \mathrm{~m}^{2}$ & $10.000 \mathrm{~m}^{2}$ \\
Área fabril & $8.500 \mathrm{~m}^{2}$ & $5.000 \mathrm{~m}^{2}$ & 760 & 160 \\
Total de postos de trabalho & 102 & 76 & 660 & \\
\hline
\end{tabular}

Fonte: documentos disponibilizados pelas empresas. 
senta a média mais elevada de NPS $(88,7 \mathrm{~dB}(\mathrm{~A}))$ e a Empresa 1, a menor média $(82,2 \mathrm{~dB}(\mathrm{~A}))$. As Empresas 1 e 2 entre si e as Empresas 3 e 4 apresentam semelhanças entre os valores das médias de NPS (teste ANOVA comparação significativa em nível 0,05 ).

A PAIR caracteriza-se por ser uma lesão auditiva irreversível. A nocividade da exposição ao ruído está relacionada com a intensidade do ruído e com o tempo de exposição, durante anos. Todos os trabalhadores analisados ficam expostos ao ruído durante uma jornada mínima de oito horas (não se computam as horas extras que por ventura realizam). Os trabalhadores foram distribuídos em função de seu histórico de tempo de serviço exposto ao ruído na Tabela 3.

A maioria dos trabalhadores tem mais de oito anos de serviço exposto ao ruído e a média é de 12,9 anos. Na análise das médias dos tempos de exposição ao ruído em cada empresa, as Empresas 3 e 4 apresentam médias semelhantes entre si e mais elevadas (14,3 e 16,0, respectivamente) do que as Empresas 1 e 2, com médias de 10,3 e 11,3, respectivamente (comparação das empresas pelo teste de Scheffé - comparação significativa em nível 0,05).

Tabela 2

Níveis de pressão sonora (NPS) e quantidade de trabalhadores expostos nas quatro empresas ( $n=741$ ).

\begin{tabular}{lcr}
\hline NPS $[\mathrm{dB}(\mathrm{A})]$ & $\mathbf{n}$ & $\%$ \\
\hline $65-83$ & 226 & 30,5 \\
$84-87$ & 220 & 29,7 \\
$88-90$ & 148 & 19,9 \\
$91-105$ & 147 & 19,8 \\
Total & 741 & 100,0 \\
\hline
\end{tabular}

Tabela 3

Tempo de serviço exposto ao ruído intenso dos trabalhadores das quatro empresas $(n=741)$.

\begin{tabular}{lc}
\hline Exposição (anos) & $\%$ \\
\hline $1-7$ & 30,0 \\
$8-13$ & 30,0 \\
$14-19$ & 20,0 \\
$>20$ & 20,0 \\
\hline
\end{tabular}

Quanto à idade desses trabalhadores, a média dos 741 trabalhadores é de 37,2 anos de idade, caracterizando um grupo não tão jovem e próximo das faixas etárias onde se iniciaria um desgaste natural da audição (ISO 1999) 19.

Na comparação das médias de idades entre os trabalhadores das quatro empresas, pela aplicação do teste de Scheffé (comparação significativa em nível 0,05), nas Empresas 3 e 4 as médias de idades são semelhantes entre si $(38,6$ e 40,6) e superiores (os trabalhadores são mais velhos) às das Empresas 1 e 2, que são semelhantes entre si $(33,1$ e 32$)$.

Alguns aspectos que relacionam as condições de ambiente de trabalho nas empresas e que são importantes como ações voltadas à preservação da audição, foram levantados e são mostrados no Tabela 4, ano a ano, no período estudado de 1997 a 2001.

Há uma intensificação na execução dos aspectos do programa preventivo nos últimos anos do período estudado nas empresas (a partir de 1999). A estruturação do PCMSO, por exemplo, é intensificada nas empresas a partir de 1999, o mesmo ocorre com o PPRA e o Mapa de Risco, que também passam a ser mais freqüentes. As orientações sobre a preservação da audição foram realizadas nas empresas nos últimos anos por ocasião da Semana Interna de Prevenção de Acidentes de Trabalho (SIPAT). Observa-se, porém, o descumprimento à legislação (NR-7) em alguns dos itens investigados: as CAT por PAIR não são emitidas em nenhuma empresa, apesar dos casos de PAIR encontrados; a avaliação do ruído ambiental não é realizada conforme se alteram os ambientes de trabalho, por exemplo, na Empresa 1 que sofreu mudanças no seu "layout" e nas empresas que adotaram medidas de controle coletivo de ruído (Empresas 1 e 2) não há reavaliação dos níveis de ruído no ambiente de trabalho modificado. A introdução de medidas de controle do ruído ocorreu em três momentos em três das empresas estudadas. A realização de audiometrias anuais é freqüente com exceção da Empresa 2. O fornecimento de protetores auriculares é o único item realizado em todos os anos por todas as empresas, mas seu controle efetuado anualmente somente nas Empresas 3 e 4.

Quanto ao perfil auditivo dos trabalhadores avaliados nas empresas, a maioria apresenta audiograma sugestivo de PAIR. Os resultados das audiometrias estão distribuídos ano a ano, para o período estudado, na Tabela 5.

As empresas com audiometrias realizadas anualmente (Empresas 1, 3 e 4), não o fazem na totalidade de seus funcionários, daí a diferença no total de exames ano a ano. 
Itens do programa de preservação auditiva implantado nas empresas de 1997 a 2001.

\begin{tabular}{|c|c|c|c|c|c|c|c|c|c|c|c|c|c|c|c|c|c|c|c|c|}
\hline \multirow{2}{*}{$\begin{array}{l}\text { Aspectos do programa } \\
\text { preventivo } \\
\text { Modificações no } \\
\text { layout da fábrica }\end{array}$} & \multicolumn{4}{|c|}{ Empresa 1} & 2001 & 1997 & \multicolumn{4}{|c|}{ Empresa 2} & 1997 & \multicolumn{3}{|c|}{ Empresa 3} & 2001 & 1997 & $\begin{array}{r}\text { Em } \\
1998\end{array}$ & $\begin{array}{r}\text { npres } \\
1999\end{array}$ & $\begin{array}{l}4 \\
2000\end{array}$ & 2001 \\
\hline & & & $x$ & $x$ & $x$ & & & & & & & & & & & & & & & \\
\hline PPRA & & & $x$ & & $x$ & & & $x$ & $x$ & $x$ & & & $x$ & $x$ & $x$ & & & $x$ & $x$ & $x$ \\
\hline Avaliação ambiental & & & & & & & & $x$ & & & & $x$ & & & $x$ & & $x$ & & & \\
\hline Avaliação do ruído & & & & & & & & $x$ & & & & $x$ & & & $x$ & & & & $x$ & \\
\hline $\begin{array}{l}\text { Confecção de mapa } \\
\text { de agentes de risco }\end{array}$ & & $x$ & & $x$ & & & $x$ & & $x$ & & & & $x$ & $x$ & $x$ & & $x$ & $x$ & $x$ & $x$ \\
\hline $\begin{array}{l}\text { Medidas coletivas } \\
\text { de controle de ruído }\end{array}$ & & & $x$ & & & & & & & $x$ & & & & & & & & $x$ & & \\
\hline $\begin{array}{l}\text { Disponibilidade de } \\
\text { protetores auriculares }\end{array}$ & $x$ & $x$ & $x$ & $x$ & $x$ & $x$ & $x$ & $x$ & $x$ & $x$ & $x$ & $x$ & $x$ & $x$ & $x$ & $x$ & $x$ & $x$ & $x$ & $x$ \\
\hline $\begin{array}{l}\text { Controle de utilização } \\
\text { de protetores auriculares }\end{array}$ & & & & & $x$ & & & $x$ & $x$ & $x$ & $x$ & $x$ & $x$ & $x$ & $x$ & $x$ & $x$ & $\mathrm{X}$ & $x$ & $x$ \\
\hline PCMSO & & & $x$ & $x$ & $x$ & & & $x$ & $x$ & $x$ & & & $x$ & $x$ & $x$ & & & $x$ & $x$ & $x$ \\
\hline Realização de audiometrias & $x$ & $x$ & $x$ & $x$ & $x$ & & $x$ & & $x$ & & $x$ & $x$ & $x$ & $x$ & $x$ & $x$ & $x$ & $x$ & $x$ & $x$ \\
\hline $\begin{array}{l}\text { Encaminhamentos } \\
\text { para médico ORL }\end{array}$ & & & & & $x$ & & & & & & & & & $x$ & $x$ & & & & $x$ & $x$ \\
\hline $\begin{array}{l}\text { Ações administrativas: } \\
\text { abertura de CAT }\end{array}$ & & & & & & & & & & & & & & & & & & & & \\
\hline
\end{tabular}

$\mathrm{X}=$ presente.

Tabela 5

Resultados anuais das avaliações auditivas dos trabalhadores nas empresas de 1997 a 2001 ( $n=2.270$ ).

\begin{tabular}{|c|c|c|c|c|c|c|c|c|c|c|}
\hline \multirow{2}{*}{ Audição } & \multicolumn{2}{|c|}{1997} & \multicolumn{2}{|c|}{1998} & \multicolumn{2}{|c|}{1999} & \multicolumn{2}{|c|}{2000} & \multicolumn{2}{|c|}{2001} \\
\hline & $\mathrm{n}$ & $\%$ & $\mathrm{n}$ & $\%$ & $\mathrm{n}$ & $\%$ & $\mathrm{n}$ & $\%$ & $\mathrm{n}$ & $\%$ \\
\hline $\begin{array}{l}\text { Limiares auditivos } \\
\text { aceitáveis }\end{array}$ & 176 & 43,13 & 217 & 41,16 & 141 & 37,49 & 238 & 42,70 & 50 & 37,30 \\
\hline PAIR & 178 & 43,62 & 229 & 43,44 & 186 & 49,45 & 250 & 44,87 & 200 & 49,74 \\
\hline $\begin{array}{l}\text { Perda auditiva } \\
\text { não ocupacional }\end{array}$ & 54 & 13,23 & 81 & 15,38 & 49 & 13,02 & 69 & 12,38 & 52 & 12,93 \\
\hline Total & 408 & 100,00 & 527 & 100,00 & 376 & 100,00 & 557 & 100,00 & 402 & 100,00 \\
\hline
\end{tabular}

No monitoramento auditivo dos trabalhadores que possuem mais de um exame audiométrico realizado no período estudado, há 104 (14\%) trabalhadores com deslocamento nos limiares auditivos, caracterizando-se uma piora na audição para as freqüências 3.000, $4.000 \mathrm{e} / \mathrm{ou}$ $6.000 \mathrm{~Hz}$, de 1997 a 2001.

Destes, 38 (36,5,\%) desenvolveram PAIR (sendo a Empresa 1 com seis casos, a 2 com seis casos, Empresa 3 com16 e Empresa 4 com dez casos) e 66 (63,5\%) já portadores de PAIR, tiveram seus limiares auditivos agravados nas freqüências 3.000, 4.000 e/ ou $6.000 \mathrm{~Hz}$ (casos de agravamentos de PAIR: na Empresa 1 com 16, a Empresa 2 com dois casos, a Empresa 3 com 43 e a Empresa 4 com cinco casos). Entre os trabalhadores com deslocamento nos limiares auditivos, $77 \%$ fazem uso constante de protetores auriculares.

Há associação entre a empresa onde o trabalhador atua e a piora nos limiares $3.000 \mathrm{e} / \mathrm{ou}$ 4.000 e/ ou $6.000 \mathrm{~Hz}\left(\chi^{2}=20,4211\right.$; probabilidade $=0,0001)$. 
Esse grupo que apresentou piora nos limiares auditivos está exposto a NPS médio de $87,7 \mathrm{~dB}$ (A) (desvio padrão de 5,2) e não há diferença significativa entre o NPS médio deste grupo trabalhadores com os trabalhadores que não pioraram seus limiares auditivos $(T=-0,55$ e $\mathrm{p}>0,5817$ ).

A média de idade calculada para o último exame realizado com os trabalhadores com piora nos limiares auditivos é de 42,8 anos (de 21 a 63 anos e desvio padrão 7,42 ). $O$ tempo médio de serviço exposto ao ruído é de 17,4 anos (de 5 a 28 anos e desvio padrão de 5,74).

Já no grupo de trabalhadores com limiares auditivos estáveis, a média de idade é de 38,9 anos (de 19 a 63 anos e desvio padrão de 9,44) e a média do tempo de serviço exposto ao ruído é de 14,5 anos (de 2 a 35 anos e desvio padrão de 7,44$)$. Tanto a média da idade como a média do tempo de serviço são estatisticamente maiores entre os trabalhadores com piora nos limiares auditivos do que entre aqueles com limiares estáveis (respectivamente, $\mathrm{T}=3,93$ e p > 0,0002; $\mathrm{T}=3,82$ e $\mathrm{p}>0,0002$ ).

No grupo geral de trabalhadores portadores de PAIR (307), a média de idade é de 42,3 anos e o tempo de exposição ocupacional ao ruído, de 16,7 anos.

Os trabalhadores, na sua maioria (86\%), relatam a utilização constante do protetor auricular, apesar dos números de casos de PAIR e pioras na audição. Há associação entre a empresa e a utilização do protetor auricular $\left(\chi^{2}=\right.$ 176,9944; probabilidade $<0,0001)$. A utilização de protetores auriculares pelos trabalhadores da Empresa 4 é de $100 \%$, na Empresa 3 é de $94,2 \%$, na 2 é de $70 \%$ e na 1 é de $56,8 \%$.

Não foi realizada uma investigação detalhada sobre a qualidade dos Equipamentos de Proteção Individual (protetores auriculares), as condições da utilização, a sua correta colocação e condições de higiene, como também a sua adequação em relação às características do ambiente de trabalho, o tipo de atividade desenvolvida e a atenuação desejada do ruído, que deveriam ser efetuadas como parte do Programa de Preservação da Audição (PPA), até que medidas ambientais fossem adotadas para reduzir o ruído e efetivamente preservar a audição.

\section{Discussão}

Em relação ao sistema de produção nas empresas metalúrgicas, que se acredita reduzir os riscos ambientais para melhorar as condições de trabalho, observa-se que essas "novas" formas de gestão encontram-se parcialmente implantadas em três das empresas estudadas com coexistência de métodos ditos tradicionais, e são concordantes com as observações de Humphrey 20 , que considera nas empresas brasileiras a adoção de algumas estratégias como o "just-in-time", flexibilização do trabalho e produção em células, sem a introdução completa dos novos modelos produtivos. Estas formas de gestão parecem não ter contribuído significativamente para a melhoria das condições para garantir a audição dos trabalhadores, principalmente nas Empresas 1 e 3, com mais casos de trabalhadores apresentando piora nos limiares auditivos. A Empresa 3 é a maior do grupo estudado (Tabela 1), no entanto, em relação aos aspectos do PPA não apresenta diferenças significativas em relação às demais, porém nesta não há referência a nenhuma tentativa de medida de controle ambiental de ruído, mesmo apresentando os NPS mais elevados.

Quanto ao tempo de exposição ao ruído durante a vida laborativa, no grupo em geral estudado a média é de 12,9 anos. A literatura considera que as alterações auditivas provocadas pelo ruído atingem seu nível máximo de lesão nos primeiros 10 a 15 anos de exposição ao ruído ${ }^{3}$. Assim, é esperada uma grande ocorrência de alterações auditivas, mas também é possível que parte da população avaliada neste estudo já esteja atingindo fase mais estável.

Os documentos relativos ao PCMSO e PPRA foram estruturados pelas empresas a partir de 1998, talvez em função das alterações na legislação ocorridas em 1998 (NR-7, Portaria 19, entre outras). Outras recomendações da legislação foram desconsiderados, como a abertura de CAT, o cálculo da dose de ruído ao qual o trabalhador está exposto e a implantação de medidas de controle ambiental do ruído abrangentes (equipamentos de proteção coletiva); houve o registro do enclausuramento do motor de uma máquina (Empresa 4) e a utilização de bicos redutores de ruído de ar comprimido nas Empresas 1 e 2, mas como ações pontuais e que beneficiam postos de trabalho isoladamente, sem um maior impacto no ambiente como um todo. As empresas optam pela adoção de protetores auriculares como medida de proteção auditiva, que deve ser considerada como uma ação paliativa e não definitiva na preservação da audição. Na análise dos aspectos importantes como parte de um PPA, pode-se observar que para as empresas estudadas, a distribuição dos protetores auriculares é um aspecto valorizado como ação preventiva dos efeitos do ruído sobre a audição. No entanto, mesmo utilizando protetores auriculares ocorreram agra- 
vamentos nos limiares auditivos de trabalhadores que já apresentavam PAIR (66 trabalhadores). Há uma certa negligência em relação ao ruído, com a avaliação da exposição ao ruído realizada esporadicamente pelas empresas e, quando o fazem, é por meio de leitura instantânea, desconsiderando a variabilidade na intensidade do ruído ambiental. Não há o cálculo da dose de ruído que cada trabalhador recebe numa jornada de trabalho, impossibilitando a sua comparação com os limites de exposição recomendados pela legislação. A avaliação do ruído é realizada por setores fabris e não por posto de trabalho. A falta de precisão quanto à exposição ao ruído recebida pelos trabalhadores dificulta o planejamento e avaliação das ações de um programa preventivo, principalmente quando observa-se que nas empresas estudadas a maioria dos trabalhadores encontra-se exposto a níveis superiores a $84 \mathrm{~dB}(\mathrm{~A})$ (Tabela 2). Segundo Lutman 21, há um risco para a audição considerado leve na exposição a $80 \mathrm{~dB}$, um risco marginal a $85 \mathrm{~dB}$ e um risco considerável a partir de $90 \mathrm{~dB}$; em torno de $20 \%$ dos expostos estudados estão nesta última categoria $(19,8 \%$ expostos entre 91 a $105 \mathrm{~dB}(\mathrm{~A}))$.

Leinster et al. 9, na Inglaterra, analisaram o PCA de 48 indústrias de vários ramos de atividade e observaram que quanto ao aspecto medição e documentação sobre o ruído segundo a legislação, $40 \%$ das empresas seguiam as recomendações sobre medição do ruído; quanto ao treinamento e informações sobre preservação da audição veiculadas para trabalhadores, 50\% das empresas realizavam orientações sobre uso correto de protetores auriculares e $26 \%$ possuíam um programa de treinamento específico para o ruído; quanto às medidas adotadas pela empresa para controle do ruído, $60 \%$ das empresas introduziram medidas de proteção coletiva ao ruído.

Indulski \& Boczkowski 22, na Polônia, observaram que a maioria das ações em saúde do trabalhador são implantadas apenas pelos profissionais da saúde e que um maior envolvimento de trabalhadores e empresários deveria ser contemplado, visando a modificações nos ambientes de trabalho.

No Brasil, Guerra et al. 23 analisaram uma indústria metalúrgica no Rio de Janeiro com 194 trabalhadores, e observaram que entre as ações referentes ao PCA da empresa não havia medidas de controle coletivo do ruído, sendo o programa estruturado para a avaliação auditiva, palestras aos trabalhadores sobre cuidados com a audição, distribuição de protetores auriculares e abertura de CAT quanto necessário. A exposição individual ao ruído não foi realizada pela empresa, o que dificultou o estudo dos autores, a empresa possuía medição do ruído por leitura instantânea por setores fabris.

Dos 741 trabalhadores analisados, a maioria apresenta alterações auditivas relacionadas ao ruído do ambiente de trabalho (Tabela 5). No acompanhamento do grupo (monitoramento auditivo) por cinco anos, há 104 com piora nos seus limiares auditivos em 3.000, e/ ou 4.000 e/ou $6.000 \mathrm{~Hz}$. Diversos fatores podem contribuir para a piora auditiva além da exposição ao ruído excessivo, como o próprio desgaste natural da audição pela idade e outros fatores considerados potencializadores do dano auditivo, entre estes, os agentes químicos ototóxicos, a temperatura ambiental elevada, o esforço físico, as condições individuais de saúde etc. Este estudo não pretende esgotar todas essas possibilidades.

Neste estudo, a idade e o tempo de serviço exposto ao ruído foram fatores associados à piora nos limiares auditivos. Quanto à idade, a média nos portadores de PAIR (42,3 anos) é superior à de idade geral dos trabalhadores estudados (37,2 anos). No monitoramento auditivo, há diferenças significativas entre as médias de idade e do tempo de serviço exposto ao ruído entre os trabalhadores com limiares auditivos estáveis (média da idade 38,9; média do tempo de serviço no ruído 14,5$)$ e aqueles com piora nos limiares auditivos $(42,8 ; 17,4)$. Corso 24 estudou os efeitos da idade sobre o trabalhador exposto ao ruído e observou que os efeitos de ambas se superpõem na lesão do ouvido interno (cóclea). Quanto mais velhos e com mais tempo de exposição ao ruído a ocorrência de PAIR foi maior, evidenciando a não garantia da preservação da audição nesse grupo.

Entre as empresas estudadas, a maioria dos casos de piora nos limiares auditivos em trabalhadores já portadores de PAIR está na Empresa 1 (com 16 casos) e na Empresa 3 (com 43 casos). Entre os fatores que podem influenciar nessa diferença entre as empresas, como a idade e o tempo de serviço, pode estar a utilização correta de protetores auriculares, que se não ocorrer satisfatoriamente quanto à atenuação do ruído desejável expõe os trabalhadores aos riscos do ruído. Guerra et al. 23 encontraram associação significativa $(p<0,05)$ entre perda auditiva induzida por ruído e nível de utilização de protetores auriculares. Alguns autores 25 questionam a atenuação do ruído referida pelo fabricante para os protetores auriculares, principalmente se não forem utilizados adequadamente (colocação, prazo de trocas e condições de higiene). $\mathrm{O}$ fator idade, que contribui para o desgaste auditivo, pode influenciar a piora nas 
empresas que apresentam trabalhadores com mais anos de exposição ao ruído. Porém, outros fatores que não foram estudados, como produtos químicos ototóxicos que podem estar presentes nos ambientes de trabalho de alguma das empresas estudadas, podem influenciar na piora auditiva.

As empresas não realizavam exames audiométricos nos trabalhadores conforme a legislação, obtendo-se um total de 2.270 exames, quantidade menor do que a esperada, o que prejudica o monitoramento auditivo e as intervenções necessárias sobre o trabalhador.

Leinster et al. 9, em seu estudo, observaram quanto à realização de audiometria nos trabalhadores, que apenas $22 \%$ das 48 empresas analisadas realizavam audiometria e na maioria destas, apenas como exame na admissão do trabalhador ao emprego. Guerra et al. 23 encontraram $6,2 \%$ de trabalhadores sem audiometria na empresa estudada.

\section{Conclusão}

A legislação nas empresas estudadas não está sendo adequadamente cumprida em relação ao controle do ruído. Este fato favorece as alterações auditivas que, no entanto, apresentamse diferentemente quanto à intensidade de seu agravamento dependendo da empresa. Percebe-se um incremento nas ações a partir de 1998, gerado pelas mudanças na legislação trabalhista, mas há muito ainda a ser realizado. Falta, talvez, um maior comprometimento dos empresários com essas melhorias para que sejam efetivamente implementadas com a mesma "qualidade total" buscada para os processos produtivos.

Há diversas irregularidades quanto ao cumprimento da legislação em relação à implantação do PCMSO e do PPRA, não apresentando o PPA das empresas em completa conformidade com os padrões exigidos. As avaliações ambientais e auditivas são ainda precárias em seu monitoramento e as empresas não notificam nenhum caso de PAIR, o que compromete as estatísticas oficiais e o direcionamento de políticas públicas nessa questão.

As modernas tecnologias, quando aplicadas, não reduziram o risco da PAIR: convivem no mesmo espaço físico maquinário moderno com outros obsoletos, o que não elimina os riscos tradicionais, como o ruído, e acrescentam novos riscos. Dos trabalhadores acompanhados nas empresas, $69,5 \%$ estão expostos a níveis de pressão sonora superiores a $84 \mathrm{~dB}(\mathrm{~A})$, mesmo com a implementação de alguns processos na gestão de produção das empresas em função das exigências para aquisição de certificações internacionais na melhoria da qualidade dos produtos.

As ações consideradas como sendo de preservação da audição dos trabalhadores são predominantemente baseadas na realização de audiometrias e no fornecimento de protetores auriculares, $86 \%$ dos trabalhadores relataram a utilização constante do protetor auricular, mas ocorreram 104 trabalhadores com piora nos seus limiares auditivos. Há necessidade de uma melhor investigação sobre como os protetores auriculares estão sendo utilizados (atenuação real, colocação, higiene etc.).

A utilização de protetores auriculares enquanto medida principal no controle dos efeitos do ruído não mostrou-se suficiente para evitar o agravamento da PAIR.

Este estudo apresenta algumas limitações, como: a análise de outros fatores que também podem interferir na qualidade da audição dos trabalhadores, mas que não foram objeto deste estudo, e os dados utilizados foram coletados de documentos da própria empresa e não foram conferidos pelas pesquisadoras por meio de instrumentos próprios. Além disso, estudos em empresas que apresentem um programa preventivo em conformidade com a legislação deveriam ser realizados para utilização como referência da adequação, ou não, das medidas indicadas como de preservação da audição. 


\section{Resumo}

Neste estudo analisou-se os programas de controle do ruído em quatro metalúrgicas de Piracicaba, São Paulo, Brasil. Estudou-se 741 trabalhadores de 1997 a 2001 e analisou-se os programas, usando-se o perfil dos trabalhadores (análise de 2.270 audiometrias realizados pelas empresas) e documentos do programa de preservação da audição (PCMSO e PPRA). Desses trabalhadores, $41 \%$ apresentaram alterações auditivas (idade média de 42,3 anos; tempo médio de serviço de 16,7 anos). Em trabalhadores com mais de uma audiometria, 104 apresentaram deslocamento do limiar auditivo. Destes, 38 (36,5\%) desenvolveram PAIR e 66 (63,5\%) portadores de PAIR tiveram seus limiares auditivos agravados. Nas empresas estudadas, 69,5\% dos trabalhadores encontravam-se expostos a ruído superior a $84 \mathrm{~dB}(A)$. Conclui-se que os Programas de Preservação da Audição não estão sendo adequadamente conduzidos, a exposição ao ruído continua excessiva, com a manutenção do risco de desenvolvimento da PAIR, e as legislações existentes não são cumpridas em relação à preservação da audição.

Exposição Ocupacional; Perda Auditiva Provocada por Ruído; Saúde Ocupacional; Programa de Saúde Ocupacional

\section{Colaboradores}

C. G. O. Gonçalves executou a pesquisa, elaborou o texto preliminar, figuras e tabelas, e fez a revisão final do texto. A. M. Iguti orientou a finalização do texto.

\section{Referências}

1. Norma Regulamentadora 15. Atividades e operações insalubres, CLT Capítulo V, Título II. Diário Oficial da União 1978; 8 jun.

2. Norma Regulamentadora no 7. Programa de Controle Médico de Saúde Ocupacional. Diário Oficial da União 1994; 30 dez.

3. Comitê Nacional de Ruído e Conservação Auditiva. Boletim no 6. Recomendações mínimas para a elaboração de um PCA. São Paulo: Comitê Nacional de Ruído e Conservação Auditiva; 1999.

4. American Academy of Otolaryngology/Head and Neck Surgery Foundation. Guide for conservation of hearing in noise. Washington DC: American Academy of Otolaryngology; 1988.

5. National Institute for Occupational Safety and Health. A practical guide to effective hearing conservation programs in the workplace. Cincinnati: National Institute for Occupational Safety and Health; 1990.

6. National Institute for Occupational Safety and Health. Preventing occupational hearing loss - a practical guide. Cincinnati: National Institute for Occupational Safety and Health; 1996. (DHHS Pub. 96-110).

7. Prasher D. NoiseChem: a European commission study of the effects of solvents and noise on the audio-vestibular system. Annals: Best Practices Workshop: combined effects of chemicals and noise on Hearing. Cincinnati: Centers for Disease Control and Prevention; 2002. p. 55-7.

8. World Health Organization. International classification of impairments, disabilities and handicaps. Geneva: World Health Organization; 1980.

9. Leinster P, Baum J, Tong D, Whitehead C. Management and motivation factors in the control of noise induced hearing loss. Ann Occup Hyg 1994; 38:644-62.

10. Martinez MC. Aplicación y evaluación de una estrategia de intervención en trabajadores expuestos a niveles elevados de ruido. Salud de los Trabajadores 1997; 2:71-9.

11. Lee-Feldstein AL. Five-year follow-up study of hearing loss at several locations within a Large automobile company. Am J Ind Med 1993; 24:4154.

12. Horg OS, Raymond DM. How seriousis hearing loss among US construction workers? In: Anais do XXVII International Congress on Occupational Health [CD-ROM]. Foz do Iguaçu: International Commission on Occupational Health; 2003.

13. Andrade CRF, Schochat E. Perfil audiométrico de trabalhadores de indústrias ruidosas. In: Anais do I Encontro Nacional de Fonoaudiologia Social e Preventiva. São Paulo: Universidade de São Paulo; 1989. p. 71-81.

14. Costa EA. Classificação e quantificação das perdas auditivas em audiometrias industriais. Rev Bras Saúde Ocup 1988; 61:35-8.

15. Kwitko A, Pezzi RG. Projeto ruído. Revista CIPA 1990; 13:20-34.

16. Franco ES. Prevalência de perdas auditivas em trabalhadores no processo de admissão em empresas da região de Campinas - SP [Dissertação de Mestrado]. São Paulo: Faculdade de Educação, 
Pontifícia Universidade Católica de São Paulo; 2000.

17. Medeiros MAT. O Centro de Referências em Saúde do Trabalhador de Campinas: trajetória de uma experiência [Dissertação de Mestrado]. Campinas: Instituto de Filosofia e Ciências Humanas, Universidade Estadual de Campinas; 2001.

18. Carneiro JCD, Barros LS, Martini NAR. Characterization of noise-induced hearing loss in construction workers. In: Anais do XXVII International Congress on Occupational Health [CD-ROM]. Foz do Iguaçu: International Commission on Occupational Health; 2003.

19. International Organization for Standardization. Acoustic determination of occupational noise exposure and estimation of noise-induced hearing impairment. 2nd Ed. Geneva: International Organization for Standardization; 1990.

20. Humphrey J. L'adaptation du modèle japonais au Brésil. In: Hirata H, editor. Autour du modèle japonais. Paris: L'Harmattan; 1992. p. 237-57.
21. Indulski JA, Bocszkowski A. Occupational health education in Poland: new needs, new requirements, new programs. Int J Occup Med Environ Health 1999; 12:3-31.

22. Lutman ME. What is the risk of noise induced loss at $80,85,90 \mathrm{~dB}(\mathrm{~A})$ and above? Occup Med 2000; 50:274-5.

23. Guerra MR, Lourenço PML, Teixeira MTB, Alves MJM. Prevalence of noise-induced hearing loss in metallurgical company. Rev Saúde Pública 2005; 39:238-44.

24. Corso JF. Age correction factor in noise-induced hearing loss: a quantitative model. Audiology 1980; 19:221-32.

25. Toivonen M, Paakkonen R, Savolainen S, Lehtomaki K. Noise attenuation and proper insertion of earplugs into ear canals. Ann Occup Hyg 2002; 46:527-30.

Recebido em 03/Nov/2004

Versão final reapresentada em 04/Ago/2005 Aprovado em 22/Ago/2005 